\title{
Infrared Sensor to Predict Atmospheric Visibility and Rainfall Rate
}

\author{
Krzysztof Holejko, Tomasz Czarnecki, and Krzysztof Perlicki
}

\begin{abstract}
The presented paper describes an instrument called Aurometer which was designed for both atmospheric visibility and rainfall rate measurement. The principle of operation of Aurometer is based on determining the amount of light scattered by particles in the air that passes through the sample volume. The achieved results show that simultaneous estimation of the atmospheric visibility and rainfall rate is possible.
\end{abstract}

Keywords-light scattering, atmospheric visibility, rainfall rate, rain gauge

\section{INTRODUCTION}

$\mathbf{T}$ HE CLEAR VISIBILITY of the environment is especially important in all kinds of transport. Dense fog or rain developed along roadways may decrease the safety of motorists considerably, thus automated detectors and monitors of the visibility, fog, rain, snow or dust are important. Such detectors are also useful for monitoring the air pollution in industrial aerials [1]. Optical sensors such as transmitometers or scatterometers have been developed to measure some environmental parameter. Transmitometers are reliable but are very expensive yet, which explains why they are deployed at critical places like major airports. The reliability of scatterometers is sometimes questionable but these instruments are less expensive than transmitometer [2], [3]. In turn real time quantitative measurement of rainfall rate is needed in many civilian and military applications. Several real time rain gauges were proposed and tested [4], [5]. In this paper we propose instrument called Aurometer which was designed for both atmospheric visibility and rainfall rate measurement. Principle of operation of presented instrument is based on determining the amount of light scattered by particles in the air which passes through the sample volume. The obtained results show that simultaneous estimation of the atmospheric visibility and rainfall rate is possible.

\section{THEORETICAL BACKGROUND}

The principles of fog and rain detection are based on light propagation properties in the atmosphere, where absorption, scattering and reflection effects influence the transmitted beam.

The power loss in a absorbing and scattering environment is proportional to the input power of the beam $P_{i n}$ and the path

This paper includes the results of work carried out within the Project KBN O/N517/624229/2010 supported during 2010-2013 by the Ministry of Science in Poland.

K. Holejko, T. Czarnecki, and K. Perlicki are with Institute of Telecommunications, Warsaw University of Technology, Nowowiejska 15/19, 00-665 Warsaw, Poland (e-mail: perlicki@tele.pw.edu.pl). length of the transmission $d R$, which leads to the well known formula for the attenuation $\alpha$ of the output power beam $P_{\text {ou }}$ :

$$
\alpha=\frac{P_{o u}}{P_{\text {in }}}=e^{-b R},
$$

where $b$ is the extinction coefficient.

According to Koschmider [6] the contrast $k=\left(L_{b}-L_{o}\right) / L_{b}$ of dark objects seen against the horizon sky as a background in the foggy atmosphere and determined by the image irradiances of the object $L_{o}$ and the background $L_{b}$, changes in the same manner, as formula (1). The standard visibility $V_{n}$ is defined as a distance $R$ for the threshold of the human discrimination estimated for the contrast $k=0.02$. Now a different value of the threshold contrast is used mainly $k=0.05$. So for $k=\exp (-b R)$ and $k=0.05$ for $R=V_{n}$ one obtains $b=3 / V_{n}$ and:

$$
V_{n}=\frac{3 R}{\ln \frac{P_{\text {in }}}{P_{\text {ou }}}}=\frac{3 R}{\ln \frac{1}{k}} .
$$

By means of formula (2) the standard visibility $V_{n}$ can be estimated in two ways:

1) By measuring the attenuation of transmitted beam along the known distance $R$, what is performed by means of so called transmissometers, which give the most accurate results of measurements.

2) By measuring the contrast of a dark object located at a known distance $R$, for instance by means of the camera TV.

Another way of measuring the standard visibility is based on measurement rather the lost of transmitted power, then the power attenuation itself. The lost of transmitted power $d P=P_{i n}-P_{o u}$ is caused mainly by scattering the beam along the distance $R$. Such a whole scattered power $d P=P_{\text {in }}-P_{\text {ou }}$ according to (1) and (2) is equal to:

$$
d P=P_{\text {in }}-P_{\text {ou }}=P_{\text {in }} \frac{3 R}{V_{n}} .
$$

It is obviously difficult to collect and to measure the whole scattered power in all space directions, but it is rather possible to measure only the part of the power $P_{s}$ in a given direction. Such a power is determined by so called scattering function $\beta(\Theta)$, which determines the intensity of radiation $I$ in the given direction $\Theta$ caused by a scattering volume $d v$ illuminated by the power density $E$ :

$$
I=E \beta(\Theta) d v .
$$

The function $\beta(\Theta)$ depends on the actual standard visibility $V_{n}$ and the direction $\Theta$. We have the following $\beta(\Theta)$ values for visibilities $V_{n}$ from 1 to $20 \mathrm{~km} \mathrm{[7]:}$ 


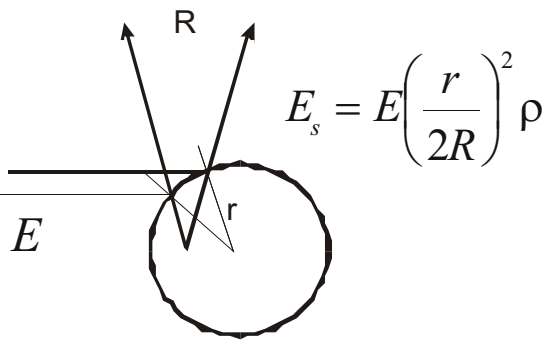

Fig. 1. Scattering from a rain drop, $\rho$ is reflection coefficient, $r$ is drop radius.

a) in forward direction (direction of transmitted beam): $\beta\left(0^{\circ}\right)=8-0.18[\mathrm{~km}]^{-1}$

b) in perpendicular direction: $\beta\left(90^{\circ}\right)=0.05-0.006[\mathrm{~km}]^{-1}$

c) in back direction: $\beta\left(180^{\circ}\right)=0.07-0.007[\mathrm{~km}]^{-1}$

d) in inclination of $40^{\circ}$ : $\beta\left(40^{\circ}\right)=0.5-0.03[\mathrm{~km}]^{-1}$

As is clearly seen from the above relationships the main part of the beam is scattered in forward direction especially for lower visibilities, while scattering for high visibility has rather spherical character. The scattered power density $E_{s}$ is proportional to the size of the drops for larger scattering particles (like rain drops). This effect is shown in Fig. 1.

\section{Aurometer}

The principle of operation of Aurometer is based on the measurement of scattered light power in the inclination of 40 degrees. The Aurometer construction is presented in Fig. 2. In turn its block diagram is shown in Fig. 3.

Our Aurometer consists of two parts: transmitting head and receiving head. Transmitting head emits IR radiation from LED1 with the wavelength of $875 \mathrm{~nm}$ and stabilized optical power. The radiation is concentrated in nearly parallel beam with the diameter of $D=25 \mathrm{~mm}$ and mean power density of $E=0.6 \mathrm{~mW} / \mathrm{cm}^{2}$. Optical radiation is modulated in square manner with the frequency of $F=2725 \mathrm{~Hz}$.

Transmitting beam intersects with the receiving beam of the same diameter. Both beams are inclined each other to about $40^{\circ}$ in order to avoid the direct coupling between the receiver

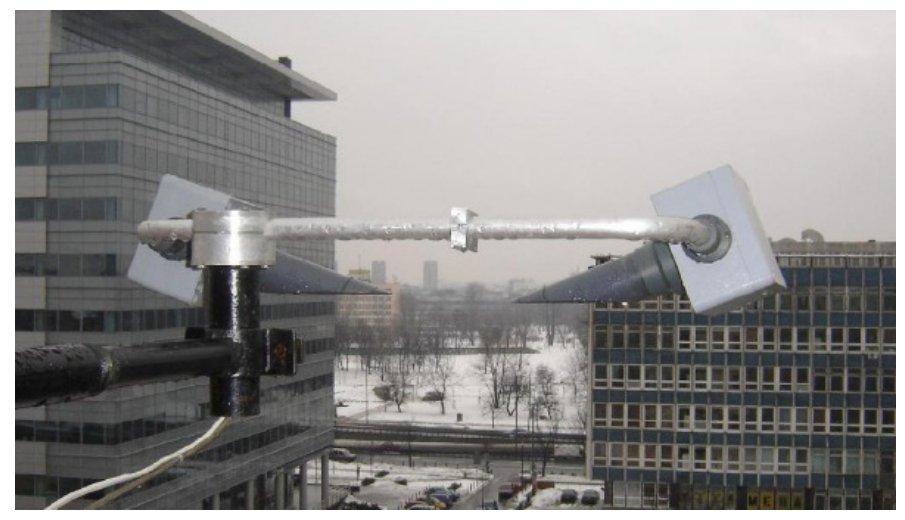

Fig. 2. Aurometer construction.

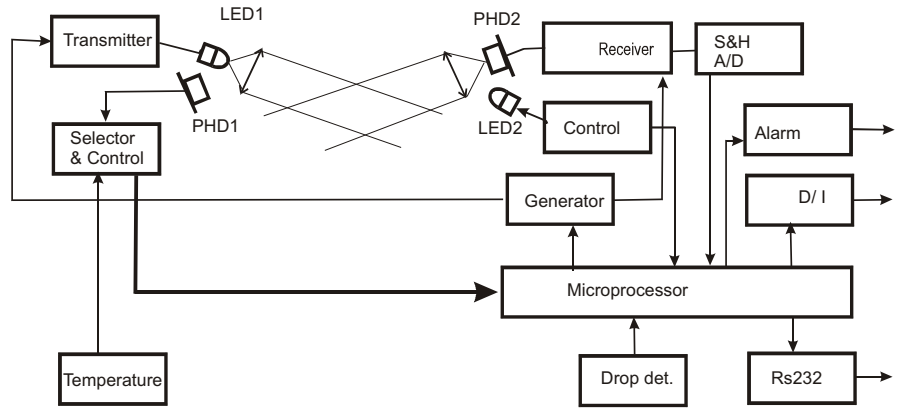

Fig. 3. Block diagram of the Aurometer; LED1, LED2 - light diodes, PHD1, PHD2 - photodiodes, Drop det. - drops sensor.

and the transmitter. The two beams form the common space volume $d v \approx 14 \mathrm{~cm}^{3}$, with the center separated from the receiving lens of $R=30 \mathrm{~cm}$.

Optical power entered on the receiving photodiode may be estimated by means of the formula (4). It should be noted that the power received by the lens with the diameter $D$ from the source with intensity of radiation $I$ is given:

$$
P_{r}=\frac{\pi D^{2}}{4} \frac{I}{R^{2}}=\frac{\pi D^{2} E \beta(\Theta)}{4 R^{2}} d v .
$$

The calculations for $D=35 \mathrm{~mm}$ show that the input power entering the photodetector for visibilities from 1 to $20 \mathrm{~km}$ are in a range of $P_{r}=(10.7-179.0) \mathrm{pW}$. The receiver contains silicon photodiode, selective low noise amplifier, homodyne mixer and a narrow band amplifier for amplifying slowly variable visibility signals and pulsed signals caused by the scattering from rain drops or snow flakes. The rain drops having the diameters from $0.7 \mathrm{~mm}$ (drizzle) to $3.0 \mathrm{~mm}$ (down pour) fall with velocities from $1 \mathrm{~m} / \mathrm{s}$ to $10 \mathrm{~m} / \mathrm{s}$. Therefore the crossing times of the vertical cross section of the space volume dv (Fig. 3) are in a range of $35.0 \mathrm{~ms}-3.5 \mathrm{~ms}$. The received pulse amplitude is proportional to the surface of the drops cross section (Fig. 3). Because of this the rainfall rate $R_{\text {rain }}[\mathrm{mm} / \mathrm{h}]$ can be estimated summing up the volume of $N$ water drops in the given time and dividing it by the horizontal cross section $A$ of the volume $d v$ :

$$
R_{\text {rain }}=\frac{\sum_{1}^{N} \frac{4}{3} \pi r_{n}^{3}}{A} .
$$

In our instrument the rain signals are measured by summing up the slope of raising rain signals in a standard interval of time.

To reduce the noise and possible cross couplings effect the receiver output signal of the receiver is measured twice. First when optical transmitter is switched on, and later when it is switched out. The output signal of the receiver is a difference of both signals. Next this signal is converted to digital form and send to the microprocessor. These both procedures are performed by the block named S\&H, A/D (Fig. 3).

Some additional blocks are used for testing and controlling the instrument (Fig. 3). First of all the clearness of lenses are tested in order to check whether they are not covered by snow or other obstacles. For that purpose the reflection from 


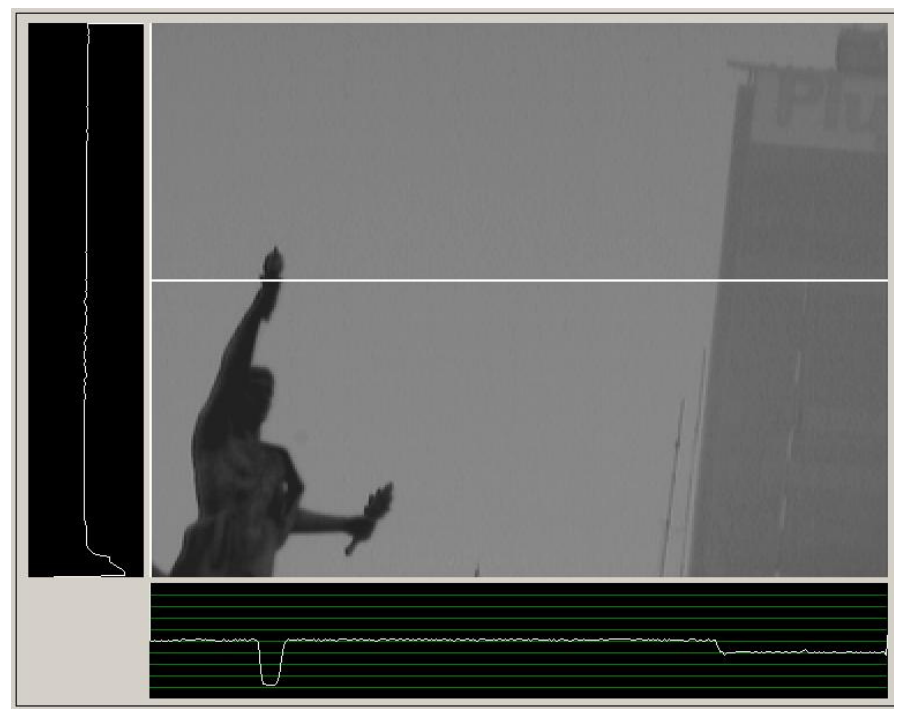

Fig. 4. Estimation of the contrast for a specific foggy scenery.

the transmitting lens is measured in the transmitter by the photodiode PHD1. Then the additional LED2 is switched on and the reflection from the receiving lens is measured. Also the additional temperature sensor and drops sensor are applied to recognize the precipitation character.

Microprocessor performs the function of controlling the system operation, testing the electronic system, calculation of output signals and generation of alarm signals. In particular microprocessor:

1 Generates the modulation signal of the frequency $F=2725 \mathrm{~Hz}$.

2 Generates the signal to control the measuring process consisting of three modes: measurement of visibility (10 s), measurement of noise and cross couplings (4 s), measurement of lens reflections (1 s).

3 Generates of test signals for testing the proper operation of the instrument. This procedure is repeated after each switching and resetting mode of the instrument.

4 Receives commands from the central computer of the net in which Aurometer is installed and elaborates of required control signals.

5 Switches on and off heating power for lenses in a case when the lens glass is bedimmed.

6 Performs suitable calculations such as mean values of visibility and precipitation within 1 minute and 1 hour.

7 Discriminates the character of precipitation basing on temperature data and drop sensor data.

8 Generates the output data via the RS 232 link and via the digital - current converter.

9 Generates threshold alarm signals.

\section{EXPERIMENTAL RESULTS}

The atmospheric visibility and rainfall rates were measured under different environment conditions. The measurement process took place: on open terrace of the six stage building, airstrip, meadow, forest. Below the atmospheric visibility and rainfall rates measurement results are presented.

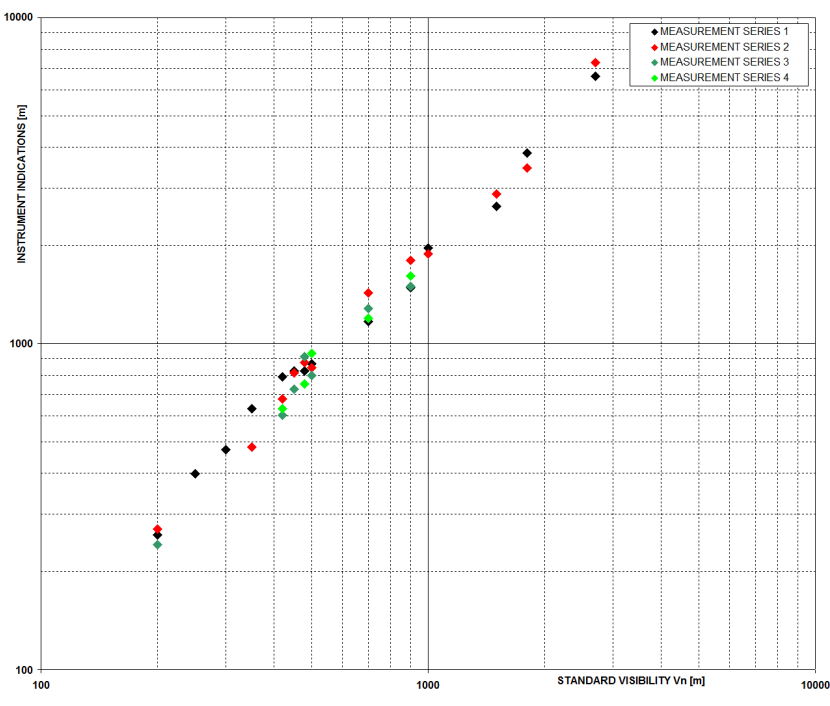

Fig. 5. Aurometer indications in different visibility conditions.

\section{A. Atmospheric Visibility}

The Aurometer atmospheric visibility was estimated by means Eq. (3). The instrument visibility indications were compared to the visual observations of different buildings situated around. The visual observations were made by TV camera. The irradiance of camera TV picture was analyzed and the distribution of the irradiance along any selected profiles was presented on the same picture (Fig. 4). Further to obtained distribution, the contrast of dark objects of known distance $R$ was estimated. Thereafter, on the basis of Eq. (2) the visual range $V_{n}$ was calculated. Figure 4 shows a dark building situated on a distance of $1200 \mathrm{~m}$. The actual so called standard visibility was equal to $V_{n}=2.7 \mathrm{~km}$.

Figure 5 presents the Aurometer indications obtained for different visibility conditions. The atmospheric visibility was in a range of $200 \mathrm{~m}$ to $2700 \mathrm{~m}$. Duration of single measurement series was 6 hours.

\section{B. Rainfall Rate}

The rainfall indication of Aurometer were compared with a real rainfall rate (Fig. 6).

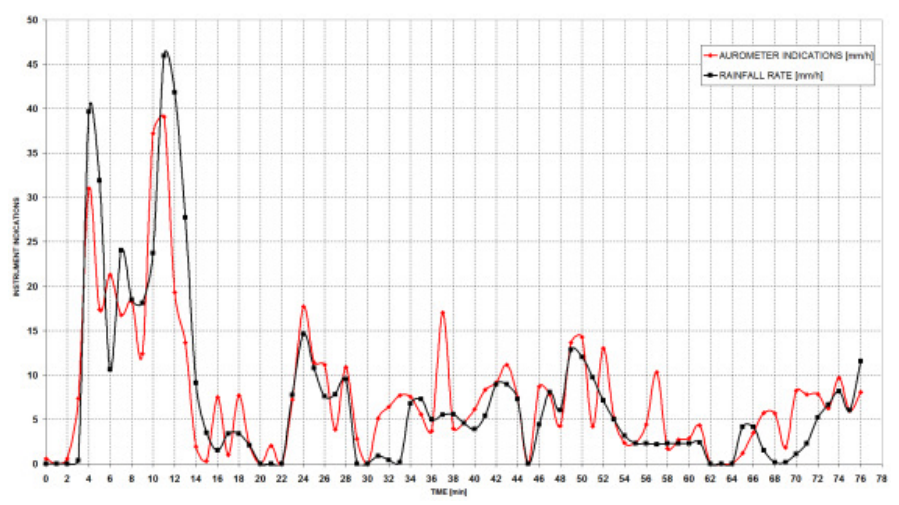

Fig. 6. Aurometer indications of rainfall rate and rain gauge results (rainfall rate). 
TABLE I

RAINFAll RATE VALUES FOR VARIOUS TyPES OF PRECIPITATION

\begin{tabular}{cccccc}
\hline \hline$<0.25$ & $0.25-0.75$ & $0.75-2.5$ & $2.5-12.5$ & $12.5-30$ & $30-100$ \\
$\mathrm{~mm} / \mathrm{h}$ & $\mathrm{mm} / \mathrm{h}$ & $\mathrm{mm} / \mathrm{h}$ & $\mathrm{mm} / \mathrm{h}$ & $\mathrm{mm} / \mathrm{h}$ & $\mathrm{mm} / \mathrm{h}$ \\
\hline drizzle & light & middle & shower & downpour & rain-storm \\
& rain & rain & of rain & & \\
\hline \hline
\end{tabular}

The Aurometer rainfall rate was calculated by means of Eq. (6). The real rainfall rate was achieved using simple rain gauge (udometer). Figure 6 shows good agreement between presented results.

Additionally, we can estimate type of precipitation based on Aurometer rainfall rate measurement.

Table I provides rainfall rate values for various types of precipitation.

\section{CONCLUSION}

Presented instrument is part of environment measurement system for assessment of weather i.e. rainfall rate, atmospheric visibility, atmospheric phenomenon (fog, rain, snow). The preliminary measurements prove the possibility of simultaneous estimation of the atmospheric visibility and rainfall rate, which is important in various means of transportation and navigation. The atmospheric visible measurements show that our instrument estimates visibility to $2700 \mathrm{~m}$. We can observed a good agreement between Aurometer indications of rainfall rate and results which were achieved using rain gauge.

\section{REFERENCES}

[1] K. Holejko and R. Nowak, "Fog detection and visibility measurements using forward scattering radiation," Opto-Electronics Review, vol. 2, pp. 107-110, 1997.

[2] R. Babari, N. Hautiere, and J. Papelard, "Computer Vision for the Remote Sensing of Atmospheric Visibility," in IEEE International Conference on Computer Vision Workshops, Barcelona, Spain, 2011, pp. 219-226.

[3] R. Siikamaki, "New product family of Present Weather Detectors and Visibility Sensors," Vaisala News, vol. 164, pp. 32-33, 2004.

[4] M. Kemp, A. Kruger, and J. Niemeier, "Design considerations and signal processing for a microwave rain gauge sensor," in IEEE International Conference on Electro/Information Technology, Indianapolis, USA, 2012, pp. $1-4$.

[5] L. Kazovsky, "An Optical Rain Gauge Based on Forward Scattering," IEEE Transactions on Geoscience and Remote Sensing, vol. 2, pp. 124131, 1985.

[6] W. Middleton, Vision through the atmosphere. Toronto: University of Toronto Press, 1952.

[7] H. Voigt, "Visibility Measurements Using Backscattered Light," Journal of the Atmospheric Sciences, vol. 5, pp. 912-918, 1968. 